\title{
A NOTE ON BALANCED-BUDGET INCOME TAXES AND AGGREGATE (IN)STABILITY IN MULTI-SECTOR ECONOMIES
}

\author{
Nicolas Abad \\ Normandie Univ., UNIROUEN, CREAM
}

\begin{abstract}
Alain Venditti
Aix-Marseille Univ., CNRS, EHESS, Centrale Marseille, AMSE and EDHEC Business School
\end{abstract}

We examine the impact of balanced-budget labor income taxes on the existence of expectation-driven business cycles in a two-sector version of the Schmitt-Grohé and Uribe (SGU) [(1997) Journal of Political Economy 105, 976-1000] model with constant government expenditures and counter-cyclical taxes. Our results show that the destabilizing impact of labor income taxes strongly depends on the capital intensity difference across sectors. Local indeterminacy is indeed more likely when the consumption good sector is capital intensive, as the minimal tax rate decreases, and less likely when the investment good sector is capital intensive, as the minimal tax rate increases. The implication of this result can be quantitatively significant. Indeed, when compared to SGU, local indeterminacy can be either completely ruled out for all OECD countries when the investment good is sufficiently capital intensive or drastically improved, delivering indeterminacy for a larger set of OECD countries, if the consumption good is sufficiently capital intensive. Focusing however on recent estimates of the sectoral capital shares corresponding to the empirically plausible case of a capital intensive consumption good, we find that there is a significant increase of the range of economically relevant labor tax rates (from a minimum tax rate of $30 \%$ to $24.7 \%$ for which local indeterminacy arises with respect to the aggregate formulation of SGU.

Keywords: Aggregate (In)stability, Local Indeterminacy, Expectations-Driven Fluctuations, Labor Income Taxes, Balanced-Budget Rule, Infinite-Horizon Two-Sector Model, Capital Intensity Difference

\section{INTRODUCTION}

Since the last financial crisis and the difficulty to control public deficit and public debt, a large debate has focused on the necessity to impose balanced-budget

This work was supported by the French National Research Agency Grants ANR-08-BLAN-0245-01 and ANR-17EURE-0020. We thank the guest editor L. Gori and two anonymous referees together with F. Dufourt, K. Gente and T. Seegmuller for useful comments and suggestions. Address correspondence to: Nicolas Abad, 3 Avenue Pasteur, 76000 Rouen, France. e-mail: nicolas.abad@univ-rouen.fr. 
rules to governments. But as initially shown by Schmitt-Grohé and Uribe (SGU) (1997), ${ }^{1}$ such a rule is likely to amplify business cycles and instability by generating expectation-driven fluctuations. SGU in particular focus their analysis on constant government expenditures financed from distortionary labor income taxes in a standard aggregate infinite-horizon model. The balanced-budget rule implies that the tax rate is endogenous, non-linear and counter-cyclical with respect to its tax base. The distortionary nature of the tax rate is related to the fact that households consider it as given. SGU then find empirically plausible conditions justifying the existence of local indeterminacy and stationary sunspot equilibria in the US economy. A simple intuition can explain this result. Suppose that agents expect an increase of the future labor tax rate. For any given capital stock, this implies a decrease of future hours worked and thus of the expected rental rate of capital. Investment and thus the current labor supply are decreased leading to a fall of current output. The labor income tax rate being counter-cyclical, the current tax rate increases, justifying the initial expectation which is then self-fulfilling.

In a two-sector model, there is no longer any perfect substitution between consumption and investment. Moreover, a two-sector formulation generates two types of amplification effects: a real effect through the Rybczynski theorem showing that an increase in the quantity of a factor leads to a more than proportional increase of the output of the good that uses that factor intensively and a price effect through the Stolper-Samuelson theorem showing that an increase in the price of one sector's output leads to a more than proportional increase in the price of the factor used intensively in that sector. The previous mechanism occurring in an aggregate setting may thus be strongly amplified or mitigated by price effects, and the plausibility of the conditions leading to the existence of expectationdriven fluctuations affected as a result. Our aim in this paper is then to study the existence of local indeterminacy under labor income taxes in a two-sector version of the SGU model as a function of the capital intensity difference across sectors. ${ }^{2}$ The question is to know whether the conditions provided by SGU in the aggregate formulation are improved or worsened by disaggregating the economy between two sectors. It is indeed fundamental (from a policy design perspective) to have the best understanding of the role of indeterminacy for business cycle fluctuations in a large class of models. ${ }^{3}$ While modern macroeconomic theory relies a lot on aggregate models, multi-sector formulations have recently been increasingly considered in the literature as they provide additional important mechanisms to explain macroeconomic instability. ${ }^{4}$

Considering two-sector models under balanced-budget rules has already appeared as providing a source of additional mechanisms increasing the occurrence of aggregate instability. Indeed in her seminal contribution, Giannitsarou (2007) raised an important debate with respect to SGU showing that if consumption taxes are used instead of labor income taxes to finance government expenditures, the steady state remains a saddle-point and local indeterminacy does not arise. The key policy implication derived by Giannitsarou is then that endogenous fluctuations under a balanced-budget rule can be avoided when consumption taxes are mixed with labor income taxes. 
Since the introduction of a consumption or a labor income tax affects in a similar way the consumption-leisure trade-off, the Giannitsarou "surprising" result has suggested the necessity to study the robustness of this no-indeterminacy conclusion, in particular, in a multi-sector version of the economy. ${ }^{5}$ Nishimura et al. (2013) have shown that the consideration of a two-sector structure has a dramatic impact on the local stability properties of the steady state. Indeed, no matter the sign of the capital intensity difference across the two sectors and still using the preference formulation of Giannitsarou (2007), expectation-driven fluctuations easily occur under consumption taxes, even in the case where both sectors have the same capital share which is usually considered to be equivalent to the aggregate formulation. Actually, in a two-sector setting with non-linear endogenous consumption taxes considered as externalities, a price distortion between consumption and investment occurs through the Stolper-Samuelson theorem and amplifies the impacts of tax expectations on production decisions leading to self-fulfilling beliefs and sunspot fluctuations.

In this paper, our main results also exhibit a strong but more complex effect of price distortions on the existence of aggregate instability under balancedbudget labor income taxes. Considering the same fundamentals' formulation as in SGU but plugged into a two-sector model, we show that local indeterminacy is obtained for both sectoral capital intensity configurations but appears to be more likely when the consumption good sector is capital intensive, as the minimal labor income tax rate above which local indeterminacy occurs is decreased, and less likely in the converse case with a capital intensive investment good sector, as the minimal labor income tax rate increases. The implication of this result can be quantitatively significant. Indeed, when compared to SGU, local indeterminacy can be either completely ruled out for all OECD countries when the investment good is sufficiently capital intensive or drastically improved, delivering indeterminacy for a larger set of OECD countries, if the consumption good is sufficiently capital intensive. Focusing however on recent estimates of the sectoral capital shares corresponding to the empirically plausible case of a capital intensive consumption good, we find that there is a significant increase of the range of economically relevant labor tax rates (from a minimum tax rate of $30 \%$ to $24.7 \%$ ) for which local indeterminacy arises with respect to the aggregate formulation of SGU. As it is standard in two-sector models, the intuition for these results relies on the differentiated impacts on relative prices and outputs of an initial expectation of increasing labor income tax rate depending on the capital intensity difference across sectors. Our conclusions therefore suggest that the design of a tax policy under a balanced-budget rule has to be carefully determined from a precise knowledge of the sectoral capital and labor shares.

The rest of the paper is organized as follows. Section 2 presents the model and the intertemporal equilibrium. In Section 3, we discuss the existence and multiplicity of steady states through the Laffer curve, we normalize the reference steady state located in the increasing part of the Laffer curve and we provide the characteristic polynomial. Section 4 contains our main results and provides economic intuitions. Section 5 discusses the empirical relevance of our conclusions 
comparing the conditions for local indeterminacy to recent estimates of labor income tax rates for the main OECD countries. Section 6 concludes, and all the proofs are gathered in the Appendix.

\section{THE MODEL}

\subsection{The Production Structure}

We consider an economy producing a consumption good $y_{0}$ and a capital good $y$. Each good is produced by capital $k_{j}$ and labor $l_{j}, j=0,1$, through a Cobb-Douglas production function. The representative firm in each industry indeed faces the following technology:

$$
y_{0}=k_{0}^{\alpha} l_{0}^{1-\alpha}, \quad y=k_{1}^{\beta} l_{1}^{1-\beta}
$$

with $\alpha, \beta \in(0,1)$. A firm in each industry maximizes its profit given the price of the consumption good (the numeraire) which is normalized to 1 , the price of the investment (capital) good $p$, the rental rate of capital $r$ and the wage rate $w$. The first order conditions give

$$
\begin{gathered}
k_{0} / y_{0}=\alpha / r \equiv a_{10}(r), l_{0} / y_{0}=(1-\alpha) / w \equiv a_{00}(w), \\
k_{1} / y=p \beta / r \equiv a_{11}(r, p), l_{1} / y=p(1-\beta) / w \equiv a_{01}(w, p) .
\end{gathered}
$$

We call $a_{i j}$ the input coefficients.

Considering that total labor is given by $\ell=l_{0}+l_{1}$, and the total stock of capital is given by $k=k_{0}+k_{1}$, the factor market clearing equation is directly obtained from the input coefficients as defined by (2). We get

$$
\left(\begin{array}{cc}
a_{00}(w) & a_{10}(r) \\
a_{01}(w, p) & a_{11}(r, p)
\end{array}\right)\left(\begin{array}{c}
y_{0} \\
y
\end{array}\right) \equiv A(w, r, p)\left(\begin{array}{c}
y_{0} \\
y
\end{array}\right)=\left(\begin{array}{l}
\ell \\
k
\end{array}\right) .
$$

From (2), substituting the expressions of $\left(k_{j}, l_{j}\right), j=0,1$ into the production functions (1) and solving with respect to $p$ gives the factor-price frontier, which provides a relationship between input prices and the capital output price. We get

$$
\left(\begin{array}{l}
1 \\
p
\end{array}\right)=A^{\prime}(w, r, p)\left(\begin{array}{c}
w \\
r
\end{array}\right) \text {. }
$$

It follows from (3) and (4) that at the equilibrium, the wage rate and the rental rate are functions of the price of the capital good only, that is $w=w(p)$ and $r=$ $r(p)$, while outputs are functions of the capital stock, total labor and the price of the capital good, $y_{0}=\tilde{y}_{0}(k, \ell, p)$ and $y=\tilde{y}(k, \ell, p)$.

Profit maximization in both sectors gives demands for capital and labor as linear homogeneous functions of the capital stock, the output of the investment good and total labor, namely $\tilde{k}_{j}=k_{j}(k, y, \ell), \tilde{l}_{j}=l_{j}(k, y, \ell), j=0,1$. The production frontier is then

$$
y_{0}=T(k, y, \ell)=\tilde{k}_{0}^{\alpha} \tilde{l}_{0}^{1-\alpha} .
$$


Note that $T(k, y, \ell)$ is a linear homogeneous function. From the envelope theorem, we get $r=\partial T / \partial k \equiv T_{1}(k, y, \ell), p=\partial T / \partial y \equiv-T_{2}(k, y, \ell)$ and $w=\partial T / \partial \ell \equiv$ $T_{3}(k, y, \ell){ }^{6}$

\subsection{Government}

In our simple neoclassical economy, as in SGU, the only source of government revenue is a labor income tax supported by the households, government purchases are constant and neither affect the consumers' preferences nor the production function, the initial stock of public debt is zero, and the government is subject to a balanced-budget requirement. The level of public spending $\mathcal{G}$ satisfies the following standard national income identity

$$
c(t)+p(t) i(t)+\mathcal{G}=y_{0}(t)+p(t) y(t)
$$

with the gross investment $i(t)=\dot{k}(t)+\delta k(t)$ and $\delta \geq 0$ the rate of depreciation of capital. However, in order to simplify the analysis, we assume that the government spending is used to produce a public good that is consumed by the households, possibly providing utility in an additively separable way, and thus does not affect the dynamic properties of the equilibrium. ${ }^{7}$ As a result, the government spending is stated in terms of the consumption good and satisfies

$$
y_{0}(t)=c_{t}+\mathcal{G},
$$

so that the investment good is as usual equal to private investment

$$
y(t)=i(t)=\dot{k}(t)+\delta k(t) .
$$

The government expenditure is equal to the total tax revenue $\Omega(t)$ generated by the tax rate $\tau$ applied to labor income $w(t) \ell(t)$ following the balanced-budget rule:

$$
\mathcal{G}=\Omega(t)=\tau w(t) \ell(t) .
$$

The government spending being constant, the tax rate is actually endogenous and satisfies

$$
\tau(t)=\frac{\mathcal{G}}{w(t) \ell(t)} .
$$

As in SGU, it is therefore counter-cyclical with respect to its tax base.

\subsection{Households' Behavior}

The economy is populated by a large number of identical infinitely lived agents. We assume without loss of generality that the total population is constant and normalized to one. At each period, a representative agent supplies elastically an amount of labor $\ell \in(0, \bar{\ell})$, with $\bar{\ell}>1$ his time endowment. He derives utility from consumption $c$ and leisure $\mathcal{L}=\bar{\ell}-\ell$ according to an additively separable function $U(c, B \mathcal{L})$, where $B>0$ is a scaling parameter, such that

$$
U(c, B \mathcal{L})=\log c-B(\bar{\ell}-\mathcal{L}) .
$$


We consider the same simple formulation as in SGU with a log-linear utility function characterized by a unitary elasticity of intertemporal substitution (EIS) in consumption and indivisible labor as in Hansen (1985).

We assume as in SGU that the representative household considers as given the whole path of the labor income tax rate which then acts as an externality. The intertemporal optimization problem is given by:

$$
\begin{aligned}
\max _{\{c(t), y(t), \ell(t)\}} & \int_{0}^{+\infty}[\log c(t)-B \ell(t)] e^{-\rho t} d t, \\
\text { s.t. } & c(t)=T(k(t), y(t), \ell(t))-\tau(t) w(t) \ell(t), \\
& \dot{k}(t)=y(t)-\delta k(t), \\
& k(0) \text { and }\{\tau(t)\}_{t \geq 0} \text { given, }
\end{aligned}
$$

where $\rho \geq 0$ is the discount rate.

\subsection{Intertemporal Equilibrium}

The Hamiltonian in current value is given by:

$$
\begin{aligned}
\mathcal{H}= & \log c(t)-B \ell(t)+\lambda(t)[T(k(t), y(t), \ell(t))-\tau(t) w(t) \ell(t)-c(t)] \\
& +q(t)[y(t)-\delta k(t)]
\end{aligned}
$$

with $q(t)$ the co-state variable which corresponds to the utility price of the capital good in current value and $\lambda(t)$ the Lagrange multiplier associated with the government budget constraint. The first order conditions of problem (10) are given by the following equations:

$$
\begin{aligned}
\frac{1}{c(t)} & =\lambda(t), \\
B & =\lambda(t) w(t)(1-\tau(t)), \\
q(t) & =p(t) \lambda(t), \\
\dot{q}(t) & =(\delta+\rho) q(t)-r(t) \lambda(t) .
\end{aligned}
$$

Mixing equations (11) and (12) gives

$$
B c(t)=(1-\tau(t)) w(t) .
$$

As shown in Section 2.1, we have $w=w(p) r=r(p), y=\tilde{y}(k, \ell, p)$ and $y_{0}=\tilde{y}_{0}(k, \ell, p)=T(k, \tilde{y}(k, \ell, p), \ell)$. Therefore, considering $c=\tilde{y}_{0}(k, \ell, p)-\mathcal{G}$ and $\tau(t)=\mathcal{G} /(w(t) \ell(t))$ into equation (15) that describes the labor-leisure trade-off at the equilibrium, we express the labor supply as a function of the capital stock and the output price, $\ell=\ell(k, p)$. Then, we get $y_{0}=y_{0}(k, p) \equiv \tilde{y}_{0}(k, \ell(k, p), p)$, $y=y(k, p) \equiv \tilde{y}_{1}(k, \ell(k, p), p)$ and thus $c=y_{0}(k, p)-\mathcal{G}$.

Considering (7) and (11)-(14), the equations of motion are finally derived as

$$
\begin{aligned}
\dot{k} & =y(k, p)-\delta k, \\
\dot{p} & =\frac{(\delta+\rho) p-r(p)+\frac{p}{c} \frac{\partial c}{\partial k}[y(k, p)-\delta k]}{E(k, p)}
\end{aligned}
$$


with

$$
E(k, p)=1-\frac{p}{c} \frac{\partial c}{\partial p} .
$$

Any solution $\{k(t), p(t)\}_{t \geq 0}$ that satisfies the transversality condition

$$
\lim _{t \rightarrow+\infty} e^{-\rho t} \lambda(t) p(t) k(t)=0
$$

is called an equilibrium path.

\section{STEADY STATE AND CHARACTERISTIC POLYNOMIAL}

\subsection{Multiplicity of Steady States and Laffer Curve}

A steady state is defined as a pair $\left(k^{*}, p^{*}\right)$ solution of the dynamical system (16):

$$
y(k, p)=\delta k, r(p)=(\delta+\rho) p .
$$

Let us denote $\kappa=k / \ell$ the capital-labor ratio. We can prove in a first step:

PROPOSITION 1. There exist unique values of $\kappa^{*}$ and $p^{*}$ such that $y\left(k^{*}, p^{*}\right)=$ $\delta \ell^{*} \kappa^{*}, r\left(p^{*}\right)=(\delta+\rho) p^{*}$.

Proof. See Appendix A.1.

However, given $\kappa^{*}$ and $p^{*}$, nothing guarantees the uniqueness of $\ell^{*}, c^{*}=c\left(\ell^{*}\right)$ and $k^{*}=k\left(\ell^{*}\right)$. The multiplicity of steady states is actually related to the existence of a Laffer curve. Using the balanced-budget rule (8) and considering given $\kappa^{*}$ and $p^{*}$, a steady state can be defined by a solution $\left(\tau^{*}, \ell^{*}\right)$ satisfying:

$$
\begin{aligned}
\mathcal{G} & =\tau^{*} w\left(p^{*}\right) \ell^{*}, \\
B c^{*} & =\left(1-\tau^{*}\right) w\left(p^{*}\right) .
\end{aligned}
$$

We then derive the following multiplicity result:

PROPOSITION 2. For given $\kappa^{*}$ and $p^{*}$, there exist $\hat{\tau} \in(0,1)$ and $\hat{\mathcal{G}}>0$ such that there are two steady states $\left(\tau^{*}, \ell^{*}\right)$ and $\left(\tau^{* *}, \ell^{* *}\right)$ such that $0<\tau^{*}<\hat{\tau}<$ $\tau^{* *}<1$ if and only if $\mathcal{G} \in(0, \hat{\mathcal{G}})$.

As illustrated on Figure 1, it follows as in SGU that there exist two steady states provided government spending is not too large, that is $\mathcal{G}=\tilde{\mathcal{G}}<\hat{\mathcal{G}}$.

Note that $\tau^{*}$ belongs to the increasing part of the curve while $\tau^{* *}$ is in the decreasing part.

\subsection{A Normalized Steady State}

We use now the scaling parameter $B>0$ in order to ensure the existence of a normalized steady state (NSS), such that $\ell^{*}=1, k^{*}=\kappa^{*}$ and $c^{*}=c(1)$, which is associated with the tax rate $\tau^{*}$ located in the increasing part of the Laffer curve. 


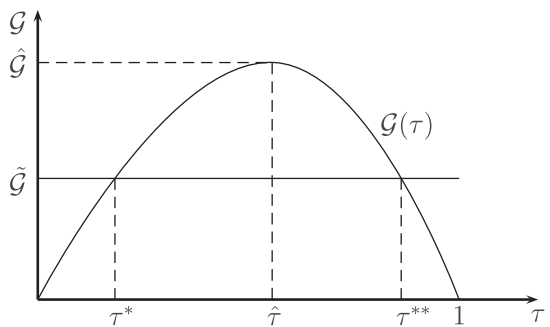

FIGURE 1. Laffer curve and multiple steady states.

PROPOSITION 3. Consider $\kappa^{*}$ and $p^{*}$ as given by Proposition 1. Then there exist $\overline{\mathcal{G}}>0$ and $B^{*}(\tau)>0$ such that when $\mathcal{G} \in(0, \overline{\mathcal{G}}),\left(k^{*}, c^{*}, \ell^{*}\right)=\left(\kappa^{*}, c(1), 1\right)$ is a NSS associated with the stationary tax rate $\tau=\tau^{*} \in(0, \hat{\tau})$ if and only if $B=$ $B^{*}\left(\tau^{*}\right)$.

Proof. See Appendix A.3.

Remark 1. Using a continuity argument, we derive from Proposition 3 that there exists an intertemporal equilibrium for any initial capital stock $k(0)$ in the neighborhood of $\kappa^{*}$.

In the rest of the paper, we will exclusively focus on the empirically relevant case where the NSS is located in the increasing part of the Laffer curve considering that $\tau<\hat{\tau}$.

\subsection{Characteristic Polynomial}

Linearizing the dynamical system (16) around the NSS gives:

$$
J=\left(\begin{array}{cc}
\frac{\partial y}{\partial k}-\delta & \frac{\partial y}{\partial p} \\
\frac{p^{*}}{c^{*}} \frac{\partial c}{\partial k}\left(\frac{\partial y}{\partial k}-\delta\right) & \frac{\delta+\rho-\frac{\partial r}{\partial p}+p^{*} \frac{\partial c}{\partial k} \frac{\partial y}{\partial p}}{E\left(k^{*}, p^{*}\right)}
\end{array}\right) .
$$

Any solution of (16) that converges to the NSS satisfies the transversality condition (18) and is an equilibrium. Therefore, given $k(0)$, if there is more than one initial price $p(0)$ in the stable manifold of the NSS, the equilibrium path from $k(0)$ will not be unique. In particular, if $J$ has two eigenvalues with negative real parts, there will be a continuum of converging paths and thus a continuum of equilibria. The NSS is locally indeterminate.

The eigenvalues of $J$ are given by the roots of the characteristic polynomial

$$
\mathcal{P}(\lambda)=\lambda^{2}-\mathcal{T} \lambda+\mathcal{D}
$$

with

$$
\begin{aligned}
& \mathcal{D}=\frac{\left(\frac{\partial y}{\partial k}-\delta\right)\left(\delta+\rho-\frac{\partial r}{\partial p}\right)}{E\left(k^{*}, p^{*}\right)}, \\
& \mathcal{T}=\frac{\left(\frac{\partial y}{\partial k}-\delta\right)\left(1-\frac{p^{*}}{c^{*}} \frac{\partial c}{\partial p}\right)+\delta+\rho-\frac{\partial r}{\partial p}+\frac{p^{*}}{c^{*}} \frac{\partial c}{\partial k} \frac{\partial y}{\partial p}}{E\left(k^{*}, p^{*}\right)} .
\end{aligned}
$$


Local indeterminacy requires therefore that $\mathcal{D}>0$ and $\mathcal{T}<0$. Obviously, saddlepoint stability is obtained when $\mathcal{D}<0$ while total instability holds if $\mathcal{D}>0$ and $\mathcal{T}>0$.

\section{LOCAL (IN)DETERMINACY WITH LABOR INCOME TAXES}

Our aim is to study the possible existence of local indeterminacy, that is business cycle fluctuations based on self-fulfilling prophecies. As initiated by Benhabib and Nishimura (1985) and (1998), the analysis in two-sector models is based on capital intensity differences across sectors. From the input coefficients defined in Section 2.1, we get the following definition:

DEFINITION 1. The consumption (investment) good is capital intensive when $b=a_{00}(\beta-\alpha) /(\delta+\rho)(1-\alpha)<(>) 0$, or equivalently when $(\beta-\alpha)<(>) 0$.

It is well known that if labor income taxes are set equal to zero, the NSS is saddle-point stable for any capital intensity difference. We now study the local stability properties of the NSS under labor income taxes. We first introduce some restrictions on the main structural parameters that are in line with the empirical estimates. Following the usual practice in the real business cycle literature, we set $\rho=0.01$, implying a net annual return on capital of around $4 \%$, and $\delta=0.025$, implying a $10 \%$ annual depreciation rate of physical capital. In a two-sector model with consumption and investment, sectoral capital shares $\alpha, \beta$ are typically between $25 \%$ and $40 \%$ of GDP in industrialized economies.

ASSUMPTION 1. $\rho=0.01, \delta=0.025$ and $\alpha, \beta \in(0.25,0.4)$.

The following proposition states necessary conditions that may hold for any capital intensity configuration, and even in the limit case with identical technologies in the two sectors, that is $\beta=\alpha$. We need however to introduce the following assumption considering the additional bound $\tilde{\tau} \equiv \zeta^{*} /\left(\zeta^{*}+b c^{*}\right)$ which affects the sign of the determinant $\mathcal{D}$ and the trace $\mathcal{T}$, and the fact that an indisputable upper bound for the labor income tax rate is $50 \%$ :

ASSUMPTION 2. $\tau<\bar{\tau}$ with $\bar{\tau}=\min \{\hat{\tau}, \tilde{\tau}, 0.5\} \quad$ if $\zeta^{*}+b c^{*}>0$, or $\bar{\tau}=\min \{\hat{\tau}, 0.5\}$ if $\zeta^{*}+b c^{*} \leq 0$.

In the limit case with $\beta=\alpha$, Assumption 2 implies that $\bar{\tau}=0.5$ as in SGU. We then get:

PROPOSITION 4. Let Assumptions 1 and 2 hold, and $\mathcal{G} \in(0, \overline{\mathcal{G}})$. Then, there exists $\underline{\tau} \in(0, \bar{\tau})$ such that a necessary condition for the local indeterminacy of the NSS and the existence of expectation-driven fluctuations is given by $\tau \in(\underline{\tau}, \bar{\tau})$, with $\lim _{\alpha \rightarrow \beta} \underline{\tau}=\beta$.

Proof. See Appendix A.4.

It is worth noting that when the two sectors have identical technologies with $\beta=\alpha$, we get as in SGU that $\underline{\tau}=\beta$, that is the minimal tax rate is equal to 
the share of capital income into GDP. We may then prove the following general result:

THEOREM 1. Let Assumption 1 hold and $\mathcal{G} \in(0, \overline{\mathcal{G}})$. Then, the NSS is saddlepoint stable when $\tau \in[0, \underline{\tau})$ and locally indeterminate with the existence of expectation-driven fluctuations when $\tau \in(\underline{\tau}, \bar{\tau})$.

Proof. See Appendix A.5.

Theorem 1 holds for any sign of the capital intensity difference $(\beta-\alpha)$. But the important question is to understand how the value of the lower bound on the tax rate $\underline{\tau}$ above which local indeterminacy occurs varies as a function of $(\beta-\alpha)$. We get the following strong result:

COROLLARY 1. Let Assumptions 1 and 2 hold, and $\mathcal{G} \in(0, \overline{\mathcal{G}})$. Then, the lower bound $\tau$ decreases and local indeterminacy is more likely when the consumption good sector is capital intensive [i.e. $(\beta-\alpha)<0)]$, while the lower bound $\tau$ increases and local indeterminacy is less likely when the investment good sector is capital intensive [i.e. $(\beta-\alpha)>0$ ].

Corollary 1 implies that, with respect to the SGU formulation with $\beta=\alpha$, the range of tax rate values for which local indeterminacy arises is increased when the consumption good sector is capital intensive and reduced when the consumption good sector is labor intensive.

A simple intuition can explain this result. Let us recall as a reference point the intuition for the existence of expectation-driven fluctuations in the aggregate framework of SGU. Suppose that agents expect an increase of the future labor tax rate. For any given capital stock, this implies a decrease of future hours worked and thus of the expected rental rate of capital. Investment and thus the current labor supply are decreased leading to a fall of current output. The labor income tax rate being counter-cyclical, the current tax rate increases, justifying the initial expectation which is then self-fulfilling.

In a two-sector model, the mechanism is different and depends on the capital intensity difference across sectors. Amplification or mitigation effects can indeed occur as, beside output effects now driven by the Rybczynski theorem, there are also relative price variations from the Stolper-Samuelson theorem. Consider first the case of a capital intensive investment good sector, that is $(\beta-\alpha)>0$, and suppose that agents expect an increase of the future labor tax rate. For any given capital stock, future hours worked still decrease. The Rybczynski theorem then implies that the expected output of the investment good sector increases more than proportionally while the output of consumption good sector decreases. The associated decrease of expected consumption generates an increase of current consumption, and thus of the current consumption good output, in order to smooth utility over time. These output variations are obtained from an increase of the current labor supply. But at the same time, there is a price effect through the Stolper-Samuelson theorem. The associated decrease of the current investment good output implies a decrease of its utility price $p$ which generates a less than 
proportional increase of the current wage rate. Adding the labor and wage effects generates an increase of the current wage income $w l$ if the capital intensity difference $(\beta-\alpha)$ is large enough and thus a decrease of the current labor income tax rate, contradicting the initial expectation. Indeterminacy is then in this case less likely.

Consider finally the case of a capital intensive consumption good sector, that is $(\beta-\alpha)<0$, and suppose that agents expect an increase of the future labor tax rate, again leading, for any given capital stock, to a decrease of future hours worked. The Rybczynski theorem now implies that the expected output of the investment good sector decreases while the output of consumption good sector increases more than proportionally. As future consumption increases, current labor and consumption decisions are weakly affected. At the same time, through the Stolper-Samuelson theorem, the decrease of the investment good output now implies a decrease of its utility price $p$ and a more than proportional decrease of the wage rate that reinforces the initial labor effect. As a whole, this mechanism generates a decrease of the expected wage income $w l$ and thus an increase of the labor income tax rate, justifying the initial expectation which is then self-fulfilling. Indeterminacy is then in this case more likely.

Remark 2. Our results appear to be quite robust with respect to our simplified specification. First, it can be shown that the existence of expectation-driven fluctuations is obtained under a non-unitary EIS in consumption $\epsilon_{c}$ as long as it is contained into an intermediary interval which contains 1 , namely $\epsilon_{c} \in\left(\underline{\epsilon}_{c}, \bar{\epsilon}_{c}\right)$ with $0<\underline{\epsilon}_{c}<1<\bar{\epsilon}_{c}$. The intuition for such a conclusion is quite standard. Sunspot fluctuations require at the same time a large enough EIS to allow the representative agent to accept consumption fluctuations over time but a not too large one in order to keep the consumption variations compatible with the expectations and the equilibrium conditions.

Second, the existence of local indeterminacy also occurs if a non-trivial exogenous distribution of the government expenditure across the two sectors, such that $y_{0}(t)=c_{t}+\mu \mathcal{G}, y(t)=i(t)+(1-\mu) \mathcal{G} / p$ with $\mu \in(0,1)$, is considered. While such a more general formulation prevents from deriving clear-cut analytical results, numerical simulations clearly shows that the existence of sunspot fluctuations is obtained under basically the same parameterization of the model ${ }^{8}$

Remark 3. The existence of sunspot fluctuations in a two-sector model with a balanced-budget rule has also been studied with government expenditure financed through a consumption tax rate. Giannitsarou (2007) has initially shown that in an aggregate model with additively separable preferences, contrary to wage income taxes, a consumption tax does not destabilize the economy. Nishimura et al. (2013) have shown that such a conclusion does not survive to the consideration of a two-sector structure. Indeed, no matter the sign of the capital intensity difference across the two sectors, expectation-driven fluctuations can occur under consumption taxes and additively separable preferences. However, local indeterminacy also appears to be more likely if the consumption good sector is more capital 
intensive than the investment good sector. The intuition again relies on the price effect through the Stolper-Samuelson theorem but the result depends strongly on the value of the EIS which has to be larger than empirically relevant values.

\section{A NUMERICAL ILLUSTRATION}

Let us now provide a simple quantitative exercise comparing our results with empirically relevant labor income tax rates for the main OECD countries as provided by Mendoza et al. (1994) and (1997), and more recently by Volkerink and De Haan (2002) and McDaniel (2007). ${ }^{9}$ According to their estimates, tax rates are given in the range for each OECD countries:

TABLE 1. Labor income tax rates of OECD countries

\begin{tabular}{ll}
$\tau \in(0.15,0.19)$ & Australia, Canada, Japan, Switzerland and USA \\
$\tau \in(0.2,0.25)$ & Spain and UK \\
$\tau \in(0.26,0.3)$ & Italy, Finland and Sweden \\
$\tau \in(0.3,0.335)$ & Austria, Belgium, France, Germany and Netherlands \\
\hline
\end{tabular}

These ranges can be compared to the lower bound $\underline{\tau}$ exhibited in our results. For a given value of $\beta \in(0.25,0.4)$ and allowing $\alpha$ to vary over the same interval, numerical simulations of $\underline{\tau}$ are given in Table 2 .

TABLE 2. $\underline{\tau}$ as a function of $\beta$ and $\alpha$

\begin{tabular}{lll}
\hline & $\alpha=0.25$ & $\alpha=0.4$ \\
\hline$\beta=0.25$ & $\underline{\tau}=0.25$ & $\underline{\tau}=0.184$ \\
$\beta=0.3$ & $\underline{\tau}=0.326$ & $\underline{\tau}=0.257$ \\
$\beta=0.35$ & $\underline{\tau}=0.396$ & $\underline{\tau}=0.331$ \\
$\beta=0.4$ & $\underline{\tau}=0.457$ & $\underline{\tau}=0.4$ \\
\hline
\end{tabular}

As can be seen from line 1 in Table 2, when the consumption good sector is capital intensive with the lowest value for $(\beta-\alpha), \underline{\tau}=0.184$ and almost all OECD countries are characterized by sunspot fluctuations. On the contrary, we conclude from line 4 that when the investment good sector is capital intensive with the largest value for $(\beta-\alpha), \underline{\tau}=0.4$ and sunspot fluctuations are ruled out for all OECD countries. As a reference point, it is worth noticing that in the aggregate framework of SGU, $\underline{\tau}=0.3$, namely the aggregate share of capital income into GDP, and only half of the OECD countries are characterized by the existence of local indeterminacy. Considering a capital intensive consumption good sector then strongly improves the plausibility of sunspot fluctuations in OECD countries.

There are only few papers that study the capital intensity difference of twosector economies with consumption and investment goods, ${ }^{10}$ so that it remains 
difficult to claim that a definitive conclusion can be reached. All of them however tend to conclude that the consumption good sector is capital intensive. Valentinyi and Herrendorf (2008) in particular provide estimates of the sectoral capital shares such that $\alpha=0.35$ and $\beta=0.28$. Using these values, we find $\underline{\tau}=0.247$ implying that except for usual countries characterized by quite low labor income tax rates, more than $50 \%$ of the OECD countries we consider may be subject to expectation-driven fluctuations due to the balanced-budget rule. When compared to the conditions of SGU where $\underline{\tau}=0.3$, we find here that there is a significant increase of the range of economically relevant labor tax rates [from $\tau \in(0.3,0.5)$ to $\tau \in(0.247,0.5)]$ for which local indeterminacy arises.

\section{CONCLUDING COMMENTS}

This paper revisits the issue of aggregate instability and expectation-driven business cycles under a balanced-budget fiscal policy rule based on labor income taxes. The new feature of our approach is to consider a two-sector economy with consumption and investment goods. Our results show that the destabilizing impact of labor income taxes initially exhibited by SGU in a standard aggregate model strongly depends here on the capital intensity difference across sectors. We prove indeed that local indeterminacy is more likely when the consumption good sector is capital intensive [i.e. $(\beta-\alpha)<0$ ], as the minimal labor income tax rate above which local indeterminacy occurs decreases, and less likely when the investment good sector is capital intensive [i.e. $(\beta-\alpha)>0$ ], as the minimal labor income tax rate increases. The implication of this result can be quantitatively significant. Indeed, when compared to SGU, local indeterminacy can be either completely ruled out for all OECD countries when the investment good is sufficiently capital intensive or drastically improved, delivering indeterminacy for a larger set of OECD countries, if the consumption good is sufficiently capital intensive.

Focusing finally on estimates of the sectoral capital shares by Valentinyi and Herrendorf (2008) corresponding to the empirically plausible case of a capital intensive consumption good sector, we find that, when compared to the conditions of SGU, there is a significant increase of the range of economically relevant labor tax rates (from a minimum tax rate of $30 \%$ to $24.7 \%$ ) for which local indeterminacy arises. Our conclusions therefore suggest that the design of a tax policy under a balanced-budget rule has to be carefully determined from a precise knowledge of the sectoral capital and labor shares.

\section{NOTES}

1. See also Abad et al. (2017) for similar results under more general preferences and technologies.

2. Huang et al. (2017) also explore the impact of labor income taxes under a balanced-budget rule in a two-sector sector closed economy. However, as they are mainly interested in studying the small economy configuration, they do not provide any analysis of the conditions for local indeterminacy in terms of the capital intensity difference across sectors. 
3. Under the same preferences as in SGU, Ghilardi and Rossi (2014) consider a CES technology instead of a Cobb-Douglas and show that aggregate instability is less likely when capital and labor are weak substitutes. Considering a discrete-time formulation of the SGU model, Anagnostopoulos and Giannitsarou (2013) show that there is a large range of economically relevant labor tax rates (from $30 \%$ to $38 \%$ ) for which local indeterminacy is ruled out with respect to the continuous-time formulation.

4. See for instance Dufourt et al. (2015) for sunspot-driven business cycles, Jaimovich and Rebelo (2009) or Beaudry and Portier (2014) for news-driven business cycles.

5. Nourry et al. (2013) focus on an aggregate model with different preferences and show that consumption taxes can generate indeterminacy if the utility function is characterized by low enough income effect.

6. It is worth recalling that due to concavity of technologies, the price of the investment good is an increasing function of the investment good output, that is $\partial p / \partial y=\partial T_{2} / \partial y=-T_{22}(k, y, \ell)>0$. This property will be useful for deriving intuitions explaining our main conclusions.

7. Considering an exogenous distribution of the government expenditure across the two sectors such that $y_{0}(t)=c_{t}+\mu \mathcal{G}, y(t)=i(t)+(1-\mu) \mathcal{G} / p$ and $\mu \in(0,1)$ does not affect our main conclusions but prevent from deriving clear-cut analytical results.

8. A proof of these two statements is available upon request.

9. We consider Australia, Austria, Belgium, Canada, Finland, France, Germany, Italy, Japan, Netherlands, Spain, Sweden, Switzerland, UK and the USA for which data are available from 1950 to 2015.

10. See Baxter (1996), Valentinyi and Herrendorf (2008) and Takahashi et al. (2012).

\section{REFERENCES}

Abad, N., T. Seegmuller and A. Venditti (2017) Nonseparable preferences do not rule out aggregate instability under balanced-budget rules: A note. Macroeconomic Dynamics 21, 259-277.

Anagnostopoulos, A. and C. Giannitsarou (2013) Indeterminacy and period length under balancedbudget rules. Macroeconomic Dynamics 17, 898-919.

Baxter, M. (1996) Are consumer durables important for business cycles? Review of Economics and Statististics 78, 147-155.

Beaudry, P. and F. Portier (2014) News-driven business cycles: Insights and challenges. Journal of Economic Literature 53, 993-1074.

Benhabib, J. and K. Nishimura (1985) Competitive equilibrium cycles. Journal of Economic Theory 35, 284-306.

Benhabib, J. and K. Nishimura (1998) Indeterminacy and sunspots with constant returns. Journal of Economic Theory 81, 58-96.

Dufourt, F., K. Nishimura and A. Venditti (2015) Indeterminacy and sunspots in two-sector RBC models with generalized no-income-effect preferences. Journal of Economic Theory 157, $1056-1080$.

Giannitsarou, C. (2007) Balanced budget rules and aggregate instability: The role of consumption taxes. The Economic Journal 117, 1423-1435.

Ghilardi, M. and R. Rossi (2014) Aggregate stability and balanced-budget rules. Journal of Money, Credit and Banking 46, 1785-1807.

Hansen, G. (1985). Indivisible labor and the business cycle. Journal of Monetary Economics 16, 309-327.

Huang, K., Q. Meng and J. Xue (2017) Balanced-budget income taxes and aggregate stability in a small open economy. Journal of International Economics 105, 90-101.

Jaimovich, N. and S. Rebelo (2009) Can news about the future drive the business cycles. American Economic Review 99, 1097-1118. 
McDaniel, C. (2007) Average Tax Rates on Consumption, Investment, Labor and Capital in the OECD 1950-2003. Working Paper Arizona State University.

Mendoza, E., G. M. Milesi-Ferretti and P. Asea (1997) On the ineffectiveness of policy in altering long-run growth: Harberger's superneutrality conjecture. Journal of Public Economics 66, 99-126.

Mendoza, E., A. Razin and L. Tesar (1994) Effective tax rates in macroeconomics: Cross-country estimates of tax rates on factor incomes and consumption. Journal of Monetary Economics 34, 297-323.

Nishimura, K., C. Nourry, T. Seegmuller and A. Venditti (2013) Destabilizing balanced-budget consumption taxes in multi-sector economies. International Journal of Economic Theory 9, $113-130$.

Nourry, K., C. Nourry, T. Seegmuller and A. Venditti (2013) Aggregate instability under balancedbudget consumption taxes: a re-examination. Journal of Economic Theory 148, 1977-2006.

Schmitt-Grohé, S. and M. Uribe (1997) Balanced-budget rules, distortionary taxes, and aggregate instability. Journal of Political Economy 105, 976-1000.

Takahashi, H., K. Mashiyama and T. Sakagami (2012) Does the capital-intensity matter? Evidence from the postwar Japanese economy and other OECD countries. Macroeconomic Dynamics 16, $103-116$.

Valentinyi, A. and B. Herrendorf (2008) Measuring factor income shares at the sectoral level. Review of Economic Dynamics 11, 820-835.

Volkerink, B. and J. De Haan (2002). Tax ratios in macroeconomics: Do taxes really matter. Empirica 29, 209-224.

\section{PROOFS}

\section{A.1. PROOF OF PROPOSITION 1}

Maximizing profit subject to the private technologies (1) gives the first order conditions

$$
\begin{aligned}
& \alpha y_{0} / k_{0}=r,(1-\alpha) y_{0} / l_{0}=w, \\
& p \beta y / k_{1}=r, p(1-\beta) y / l_{1}=w .
\end{aligned}
$$

Considering the steady state with $y^{*}=\delta k^{*}$ and $\left.r^{*} \delta+\rho\right) p^{*}$, we get

$$
k_{1}^{*}=\frac{\delta \beta}{\delta+\rho} k^{*} .
$$

Using the production function (1) for the investment good, we derive

$$
l_{1}^{*}=\left(\frac{\beta}{\delta+\rho}\right)^{-\frac{\beta}{1-\beta}} \delta k \text { and thus } \frac{k_{1}^{*}}{l_{1}^{*}}=\left(\frac{\beta}{\delta+\rho}\right)^{\frac{1}{1-\beta}} .
$$

Finally, we obtain from (A.1):

$$
\frac{\alpha(1-\beta)}{\beta(1-\alpha)}=\frac{l_{1}^{*} k_{0}^{*}}{k_{1}^{*} l_{0}^{*}} \Leftrightarrow \frac{k_{0}^{*}}{l_{0}^{*}}=\frac{\alpha(1-\beta)}{\beta(1-\alpha)}\left(\frac{\beta}{\delta+\rho}\right)^{\frac{1}{1-\beta}} .
$$


Considering (A.3), (A.4) and $l_{0}^{*}+l_{1}^{*}=\ell^{*}, k^{*}=k_{0}^{*}+k_{1}^{*}$, we get

$$
k^{*}=\frac{\frac{\alpha(1-\beta)}{\beta(1-\alpha)}\left(\frac{\beta}{\delta+\rho}\right)^{\frac{1}{1-\beta}}}{1-\frac{\beta \delta}{\delta+\rho}\left[1-\frac{\alpha(1-\beta)}{\beta(1-\alpha)}\right]} \ell^{*} \equiv \kappa^{*} \ell^{*} .
$$

Equation (A.1) with (A.4) gives

$r^{*}=\alpha\left(\frac{\alpha(1-\beta)}{\beta(1-\alpha)}\right)^{-(1-\alpha)}\left(\frac{\beta}{\delta+\rho}\right)^{-\frac{1-\alpha}{1-\beta}}, w^{*}=(1-\alpha)\left(\frac{\alpha(1-\beta)}{\beta(1-\alpha)}\right)^{\alpha}\left(\frac{\beta}{\delta+\rho}\right)^{\frac{\alpha}{1-\beta}}$.

Considering then the fact that $r^{*}=(\delta+\rho) p^{*}$ implies

$$
p^{*}=\frac{\alpha}{\beta}\left(\frac{\alpha(1-\beta)}{\beta(1-\alpha)}\right)^{-(1-\alpha)}\left(\frac{\beta}{\delta+\rho}\right)^{\frac{\alpha-\beta}{1-\beta}} .
$$

Moreover, using the fact that consumption is given by $c=y_{0}-\mathcal{G}$, we get after straightforward computations

$$
c^{*}=\ell^{*} \frac{\left(\frac{\alpha(1-\beta)}{\beta(1-\alpha)}\right)^{\alpha}\left(\frac{\beta}{\delta+\rho}\right)^{\frac{\alpha}{1-\beta}}\left(1-\frac{\delta \beta}{\delta+\rho}\right)}{1-\frac{\delta \beta}{\delta+\rho}\left[1-\frac{\alpha(1-\beta)}{\beta(1-\alpha)}\right]}-\mathcal{G} \equiv \ell^{*} \zeta^{*}-\mathcal{G} .
$$

For reference, note that at the steady state

$$
\begin{array}{ll}
a_{11}=\frac{\beta p^{*}}{r^{*}}=\frac{\beta}{\delta+\rho}, & a_{01}=\frac{p^{*}(1-\beta)}{w^{*}}=\left(\frac{\beta}{\delta+\rho}\right)^{\frac{-\beta}{1-\beta},} \\
a_{10}=\frac{\alpha}{r^{*}}=\left[\frac{\alpha(1-\beta)}{\beta(1-\alpha)}\right]^{1-\alpha}\left(\frac{\beta}{\delta+\rho}\right)^{\frac{1-\alpha}{1-\beta}}, & a_{00}=\frac{1-\alpha}{w^{*}}=\left[\frac{\alpha(1-\beta)}{\beta(1-\alpha)}\right]^{-\alpha}\left(\frac{\beta}{\delta+\rho}\right)^{\frac{-\alpha}{1-\beta}},
\end{array}
$$

and that

$$
\zeta^{*}=\frac{1-\delta a_{11}}{a_{00}\left(1-\delta a_{11}\right)+\delta a_{01} a_{10}}
$$

\section{A.2. PROOF OF PROPOSITION 2}

Consider equations (20) and (21) and the expressions of $\kappa^{*}$ and $p^{*}$ as given in the proof of Proposition 1. Substituting (A.7) into (21) and solving for the labor supply gives

$$
\ell=\ell(\tau) \equiv \frac{\frac{(1-\tau) w\left(p^{*}\right)}{B}+\mathcal{G}}{\zeta^{*}} \in(0, \bar{\ell})
$$


Substituting this expression into (20) and solving for $\mathcal{G}$ gives

$$
\mathcal{G}(\tau)=\frac{\tau(1-\tau) w\left(p^{*}\right)^{2}}{B\left[\zeta^{*}-\tau w\left(p^{*}\right)\right]}
$$

and we get $\mathcal{G}(\tau)=0$ when $\tau=0$ or 1 . Then there exists a unique $\hat{\tau} \in(0,1)$, namely

$$
\hat{\tau}=\frac{\zeta^{*}-\sqrt{\zeta^{*}\left[\zeta^{*}-w\left(p^{*}\right)\right]}}{w\left(p^{*}\right)}
$$

such that $\mathcal{G}^{\prime}(\hat{\tau})=0$ and thus a unique associated maximal value of $\mathcal{G}=\mathcal{G}(\hat{\tau})$ denoted $\hat{\mathcal{G}}$. It follows that for any given $\mathcal{G} \in(0, \hat{\mathcal{G}})$, there exist two steady states $\left(\tau^{*}, \ell^{*}\right)$ and $\left(\tau^{* *}, \ell^{* *}\right)$ such that $0<\tau^{*}<\hat{\tau}<\tau^{* *}<1$.

\section{A.3. PROOF OF PROPOSITION 3}

To establish the existence of the normalized steady state with $\ell^{*}=1$ associated with the stationary tax rate $\tau=\tau^{*}$ in the increasing part of the Laffer curve, we have to prove the existence and uniqueness of a solution $B^{*}\left(\tau^{*}\right)$ solving equation (21) with $w\left(p^{*}\right)=w^{*}$ as given by (A.6). Obviously we get

$$
B^{*}\left(\tau^{*}\right)=\frac{\left(1-\tau^{*}\right) w\left(p^{*}\right)}{\zeta^{*}-\mathcal{G}} .
$$

We need also to check that the steady state values of capital and consumption are positive when $\ell^{*}=1$. From (A.5) and (A.7), this is the case if

$$
\mathcal{G}<\frac{\left(\frac{\alpha(1-\beta)}{\beta(1-\alpha)}\right)^{\alpha}\left(\frac{\beta}{\delta+\rho}\right)^{\frac{\alpha}{1-\beta}}\left(1-\frac{\delta \beta}{\delta+\rho}\right)}{1-\frac{\delta \beta}{\delta+\rho}\left(1-\frac{\alpha(1-\beta)}{\beta(1-\alpha)}\right)} \equiv \mathcal{G}^{\max } .
$$

The result follows denoting $\overline{\mathcal{G}}=\min \left\{\hat{\mathcal{G}}, \mathcal{G}^{\text {max }}\right\}$.

\section{A.4. PROOF OF PROPOSITION 4}

Let $b=\left(a_{11} a_{00}-a_{10} a_{01}\right)$ the capital intensity difference across the two sectors. Using (2), we easily derive at the NSS that $b=a_{00}(\beta-\alpha) /(\delta+\rho)(1-\alpha)$ so that $(\beta-\alpha) b>0$. Total differentiation of the factor-price frontier (4) and the factor market clearing equation (3) gives at the NSS:

$$
\begin{aligned}
\frac{d r}{d p} & =\frac{a_{00}}{b}, \frac{d w}{d p}=-\frac{a_{10}}{b}, \frac{d y}{d k}=\frac{a_{00}}{b}-\frac{a_{10}}{b} \frac{d \ell}{d k}, \frac{d y_{0}}{d k}=\frac{d c}{d k}=-\frac{a_{01}}{b}+\frac{a_{11}}{b} \frac{d \ell}{d k}, \\
\frac{d y}{d p} & =\frac{a_{00}\left[(1-\alpha) \kappa^{*}+\alpha \frac{a_{10}}{a_{00}}\right]}{b(\beta-\alpha) p^{*}}-\frac{y^{*}}{p^{*}}-\frac{a_{10}}{b} \frac{d \ell}{d p}, \frac{d y_{0}}{d p} \\
& =\frac{d c}{d p}=\frac{-a_{01}\left[(1-\alpha) \kappa^{*}+\alpha \frac{a_{11}}{a_{01}}\right]}{b(\beta-\alpha) p^{*}}+\frac{a_{11}}{b} \frac{d \ell}{d p} .
\end{aligned}
$$


We finally need to compute $d \ell / d p$ and $d \ell / d k$. Let us denote

$$
g(k, p) \equiv 1-\tau=1-\frac{\mathcal{G}}{w(p) \ell(k, p)} .
$$

We easily derive the following elasticities:

$$
\epsilon_{g k}=\frac{\partial g}{\partial k} \frac{k^{*}}{g^{*}}=\frac{\tau}{1-\tau} \frac{d \ell}{d k} \frac{k^{*}}{\ell^{*}} \text { and } \epsilon_{g p}=\frac{\partial g}{\partial p} \frac{p^{*}}{g^{*}}=\frac{\tau}{1-\tau}\left(\frac{d w}{d p} \frac{p^{*}}{w^{*}}+\frac{d \ell}{d p} \frac{p^{*}}{\ell^{*}}\right) .
$$

From equation (15), tedious but straightforward computations yield

$$
\begin{aligned}
& \frac{d \ell}{d k}=\frac{\frac{a_{01}}{b c^{*}}}{\frac{a_{11}}{b c^{*}}-\frac{\tau}{1-\tau}}, \\
& \frac{d \ell}{d p}=\frac{\frac{a_{01}}{p^{*} c^{*} b(\beta-\alpha)}\left[(1-\alpha) \kappa^{*}+\frac{a_{11}}{a_{01}} \alpha\right]-\frac{a_{10}}{b w^{*}(1-\tau)}}{\frac{a_{11}}{b c^{*}}-\frac{\tau}{1-\tau}} .
\end{aligned}
$$

Consider then the factor-price frontier (4). Solving for $w$ gives:

$$
w^{*}=\frac{a_{10} p^{*}(\beta-\alpha)}{b \alpha} \Leftrightarrow \frac{a_{10}}{b w^{*}}=\frac{\alpha}{(\beta-\alpha) p^{*}} .
$$

The derivative $d \ell / d p$ in (A.12) then becomes

$$
\frac{d \ell}{d p}=\frac{\frac{a_{01}}{p^{*} c^{*} b(\beta-\alpha)}\left[(1-\alpha) k \kappa^{*}+\frac{a_{11}}{a_{01}} \alpha\right]-\frac{\alpha}{(\beta-\alpha)(1-\tau) p^{*}}}{\frac{a_{11}}{b c^{*}}-\frac{\tau}{1-\tau}} .
$$

It follows that

$$
\begin{aligned}
E\left(k^{*}, p^{*}\right) & =\frac{\frac{a_{11}}{c^{*} b} \frac{\beta}{(\beta-\alpha)}-\frac{\tau}{1-\tau}\left[1+\frac{a_{01}(1-\alpha) \kappa^{*}}{c^{*} b(\beta-\alpha)}\right]}{\frac{a_{11}-\frac{\tau}{b c^{*}}}{1-\tau}}, \\
\frac{p}{c} \frac{\partial c}{\partial k} & =\frac{\frac{\tau}{1-\tau} \frac{a_{01} p^{*}}{c^{*} b}}{\frac{a_{11}-\frac{\tau}{b c^{*}}}{1-\tau}}, \\
\frac{\partial y}{\partial p} & =\frac{\frac{\kappa^{*}}{c^{*} b(\beta-\alpha) p^{*}}\left[(1-\alpha)\left(1-\delta a_{11}\right)+\delta a_{11}(1-\beta)-\frac{\tau a_{00} c^{*}\left[(1-\alpha)^{2}(\delta+\rho)-\delta(\beta-\alpha)^{2}\right]}{(1-\tau)(\delta+\rho)(1-\alpha)}\right]+\frac{\alpha a_{10}}{b(\beta-\alpha) p^{*}}}{\frac{a_{11}}{b c^{*}-\frac{\tau}{1-\tau}},} \\
\frac{\partial y}{\partial k}-\delta & =\frac{\left[a_{00}\left(1-\delta a_{11}\right)+\delta a_{01} a_{10}\right]\left(\frac{\zeta^{*}}{b c^{*}}-\frac{\tau}{1-\tau}\right)}{\frac{a_{11}-\frac{\tau}{b c^{*}}-\tau}{1-\tau}} .
\end{aligned}
$$

Substituting (A.11), (A.12), (A.14) and (A.15) into (23) finally gives:

$$
\mathcal{D}(\tau)=-\frac{\left[a_{00}\left(1-\delta a_{11}\right)+\delta a_{01} a_{10}\right]\left[\zeta^{*}-\tau\left(\zeta^{*}+b c^{*}\right)\right]\left[a_{00}\left[1-(\delta+\rho) a_{11}\right]+(\delta+\rho) a_{10} a_{01}\right]}{a_{11} \beta-\tau\left[a_{11} \beta+c^{*} b(\beta-\alpha)+a_{01}(1-\alpha) \kappa^{*}\right]}
$$


and

$$
\begin{aligned}
\mathcal{T}(\tau)= & \frac{\left[a_{00}\left(1-\delta a_{11}\right)+\delta a_{01} a_{10}\right]\left[\zeta^{*}-\tau\left(\zeta+b c^{*}\right)\right]}{a_{11}-\tau\left(a_{11}+b c^{*}\right)} \\
& -\frac{\left[a_{00}\left[1-(\delta+\rho) a_{11}\right]+(\delta+\rho) a_{10} a_{01}\right]\left[a_{11}-\tau\left(a_{11}+b c^{*}\right)\right]}{a_{11} \beta-\tau\left[\beta a_{11}+c^{*} b(\beta-\alpha)+a_{01}(1-\alpha) \kappa^{*}\right]} \frac{(\delta+\rho)(1-\alpha)}{a_{00}} \\
& +\frac{\frac{\tau a_{01}(1-\tau)}{a_{11}-\tau\left(a_{11}+b c^{*}\right)}\left\{\kappa^{*}\left[(1-\alpha)\left(1-\delta a_{11}\right)+\delta a_{11}(1-\beta)-\frac{\tau c^{*} a_{00}}{1-\tau} \frac{(1-\alpha)^{2}(\delta+\rho)-\delta(\beta-\alpha)^{2}}{(\delta+\rho)(1-\alpha)}\right]+\alpha a_{10} c^{*}\right\}}{a_{11} \beta-\tau\left[a_{11} \beta+c^{*} b(\beta-\alpha)+a_{01}(1-\alpha) \kappa^{*}\right]} .
\end{aligned}
$$

Consider the expression of $\mathcal{D}$ evaluated at the NSS corresponding to the steady state $\tau^{*}$ in the increasing part of the Laffer curve. Focus first on the condition implying that the term $\zeta^{*}-\tau\left(\zeta^{*}+b c^{*}\right)$ is positive. There are two cases: either $b$ is sufficiently negative to imply $\zeta^{*}+b c^{*} \leq 0$ and thus $\zeta^{*}-\tau\left(\zeta^{*}+b c^{*}\right)>0$ or $b$ is positive or weakly negative to imply $\zeta^{*}+b c^{*}>0$ and $\zeta^{*}-\tau\left(\zeta^{*}+b c^{*}\right)>0$ if $\tau<\zeta^{*} /\left(\zeta^{*}+b c^{*}\right) \equiv \tilde{\tau}$. In this last case, we can easily show that under Assumption 1, $\tilde{\tau}>0.4$ a value which is too high to be empirically relevant (see Table 1 above). If $\zeta^{*}+b c^{*}>0$, we then introduce $\bar{\tau} \equiv \min \{\hat{\tau}, \tilde{\tau}\}$ and assume $\tau<\bar{\tau}$, while if $\zeta^{*}+b c^{*}<0$, we stick to the restriction $\tau<\hat{\tau}$. This leads to Assumption 2 .

Under Assumption 2, the numerator of $\mathcal{D}$ is then always positive. It follows that $\mathcal{D}>0$ if and only if its denominator is negative. To study the sign of this expression as a function of $\tau$, we need first to consider the fact that at the NSS, $c^{*}=\zeta^{*}-\mathcal{G}=\zeta^{*}-\tau w^{*}$ which implies that it is a degree-2 polynomial of $\tau$ such that

$$
\mathcal{P}(\tau)=\tau^{2} w^{*} b(\beta-\alpha)-\tau\left[a_{11} \beta+\zeta^{*} b(\beta-\alpha)+a_{01}(1-\alpha) \kappa^{*}\right]+a_{11} \beta .
$$

The associated discriminant is then

$$
\Delta=\left[a_{11} \beta+\zeta^{*} b(\beta-\alpha)+a_{01}(1-\alpha) \kappa^{*}\right]^{2}-4 a_{11} \beta w^{*} b(\beta-\alpha),
$$

which can be shown to be positive under Assumption 1. It follows that $\mathcal{P}(\tau)<0$ if and only if $\tau \in\left(\tau_{-}, \tau_{+}\right)$with

$$
\begin{aligned}
\tau_{-} & =\frac{a_{11} \beta+\zeta^{*} b(\beta-\alpha)+a_{01}(1-\alpha) \kappa^{*}-\sqrt{\Delta}}{2 w^{*} b(\beta-\alpha)} \text { and } \\
\tau_{+} & =\frac{a_{11} \beta+\zeta^{*} b(\beta-\alpha)+a_{01}(1-\alpha) \kappa^{*}+\sqrt{\Delta}}{2 w^{*} b(\beta-\alpha)} .
\end{aligned}
$$

Under Assumption 1, straightforward computations show that $\tau_{-}<\bar{\tau}<\tau_{+}$. The result then follows denoting $\underline{\tau} \equiv \tau_{-}$. Finally, a straightforward application of l'Hospital's rule leads to $\lim _{\alpha \rightarrow \beta} \underline{\tau}=\beta$.

\section{A.5. PROOF OF THEOREM 1}

Let us consider the trace $\mathcal{T}$. Obviously, we have $(1-\alpha)^{2}(\delta+\rho)-\delta(\beta-\alpha)^{2}>0$. Assumption 2 implies $\tau<\hat{\tau}$ and straightforward computations show that $a_{11}-\tau\left(a_{11}+\right.$ $\left.b c^{*}\right)>0$ and

$$
\kappa^{*}\left[(1-\alpha)\left(1-\delta a_{11}\right)+\delta a_{11}(1-\beta)-\frac{\tau c^{*} a_{00}}{1-\tau} \frac{(1-\alpha)^{2}(\delta+\rho)-\delta(\beta-\alpha)^{2}}{(\delta+\rho)(1-\alpha)}\right]+\alpha a_{10} c^{*}>0 .
$$

It is worth noting here that under $\tau<\hat{\tau}, B^{*}\left(\tau^{*}\right)>1$ and that

$$
\overline{\mathcal{G}}<\left.\mathcal{G}(\hat{\tau})\right|_{B=1}=\frac{\hat{\tau}(1-\hat{\tau}) w\left(p^{*}\right)^{2}}{\zeta^{*}-\hat{\tau} w\left(p^{*}\right)} \text {. }
$$


Using this property we get:

(i) when $\tau \in(0, \underline{\tau})$, we have $\mathcal{D}<0$ and the NSS is saddle-point stable;

(ii) when $\tau \in[\underline{\tau}, \bar{\tau})$, we have $\mathcal{D}>0, \lim _{\tau \rightarrow \underline{\tau}} \mathcal{T}(\tau)=-\infty, \lim _{\tau \rightarrow \bar{\tau}} \mathcal{T}(\tau)<0$ and $\mathcal{T}^{\prime}(\tau)>0$. It follows that $\mathcal{D}>0$ and $\mathcal{T}(\tau)<0$ for any $\tau \in[\underline{\tau}, \bar{\tau})$ and the NSS is locally indeterminate.

\section{A.6. PROOF OF COROLLARY 1}

Let Assumptions 1 and 2 hold, and $\mathcal{G} \in(0, \overline{\mathcal{G}})$. Tedious but straightforward computations allow to derive

(i) $\partial \underline{\tau} / \partial \alpha<0$ for any given $\beta \in(0.25,0.4)$,

(ii) $\partial \underline{\tau} / \partial \beta>0$ for any given $\alpha \in(0.25,0.4)$.

The result follows. 